\title{
AN INTERVAL-BASED APPROACH FOR REASONING ABOUT LAND USE CHANGE TRAJECTORIES
}

\author{
Adeline M. Maciel*, Lubia Vinhas*, Gilberto Camara*, Michelle C. A. Picoli, Rodrigo A. Begotti \\ Image Processing Division, National Institute for Space Research (INPE), \\ Av. dos Astronautas 1758, São José dos Campos, São Paulo, 12227-001, Brazil
}

\begin{abstract}
In this paper, we present a formal spatiotemporal interval logic mechanism extended from Allen's interval temporal logic to the spatial context. The method contributes with a tool to help scientists and policy makers to reason about land use change. We use an interval-based approach and the concept of events to reason about land use changes trajectories resulting in a formalism that allows users to express queries about the land use. To show the use of our formalism we use it to identify and quantifying land use transitions in Mato Grosso state in Brazil from annual classified maps of land use.
\end{abstract}

Index Terms - Spatiotemporal, Interval-based, Land use change, Trajectories, Events

\section{INTRODUCTION}

Brazil, more than any other country in the world faces the challenge of balancing agricultural production and environmental protection [1]. Historically, forest conversion has begun with small-scale exploration, as subsistence agriculture, followed by consolidation within large-scale cattle ranching operations or abandonment to secondary forest [2]. But, in the last decade, the expansion of large-scale mechanised agriculture at the forest frontier has introduced as a potential new pathway for loss of forest [2]. This has generated a debate over the impact of pasture and cropland expansion to deforestation and, consequently, on the economy [3]. Thus, we contributed to the scientific debate on the subject, as we propose methods for improving the extraction of information about land use change.

Chini and collaborators (2012) [4] defines land use transitions as annual changes between land use states, where land use states are the fractions of a given spatial unit in study occupied by different land uses in a given year. We take this def-

\footnotetext{
* Corresponding author. Email: adeline.maciel@inpe.br, lubia.vinhas @ inpe.br and gilberto.camara.inpe@gmail.com.

This work is supported by the São Paulo Research Foundation (FAPESP) e-Science Program (grant 2014/08398-6). Adeline M. Maciel is supported by the Coordination for the Improvement of Higher Education Personnel (CAPES), and Gilberto Camara is also supported by National Council for Scientific and Technological Development (CNPq) (grants 312151/2014-4).
}

inition to use the term transition as referring to changes from one class of use to another, for example when a forested area is converted to cropland. In order to observe and monitoring transitions, besides the underlying spatial support, the temporal component is also important to study land use change. According to Zhou and co-authors (2008) [5], the term trajectory refers to successions of land cover classes (e.g. forest, cropland, water) in a given spatial unit (e.g. pixels) over more than two observations at different times. For instance, during three annual observations of land parcel one found respectively to be a forest than a pasture area and finally a cropland area. So, we have a trajectory of forest $\rightarrow$ pasture $\rightarrow$ cropland with three transitions during the studied period. We consider trajectories as a sequence of transitions observed in a given unit of analysis (e.g. pixels or other elements of a spatial partition). As transitions do not follow a fixed pattern a large variability of trajectories appear, specially when observing large areas during long periods of time [6]. This work takes Zhou et al. [5] definition as a conceptual basis for developing a formalism for reasoning about land use change.

Nowadays, the main source of data to generate trajectories of change over large areas (e.g. the entire Amazon biome) and long periods of time is Earth observation (EO) satellites. They provide consistent information about the Earth's land and oceans, since their sensors observe the same area many times, at local, regional, national or global scale, producing a continuous stream of physical variables. The use of remote sensing satellite data has being of crucial importance to monitor land use change in Brazil. For example, the Program for the Estimation of Deforestation in the Brazilian Amazon (PRODES) project, conducted by the National Institute for Space Research (INPE), uses images medium resolution (Landsat, CBERS and other) to map the deforestation increments since 1988 as well as the annual deforestation rate, for the entire Brazilian Legal Amazon area [7].

Once the maps of land use are produced we still need to be able to extract the essential information from it, allowing scientist to understand the dynamics of change. This research aims to contribute with this demand by providing spatiotemporal mechanism to analyse and representation of land use changes. Thus, we explored the following question: How best to describe and reason about land use change trajectories by 
analysis on big EO data sets? Its core is an extension and improvement of the interval-based approach by Allen's [8] to build a general calculus to reason about land use change trajectories, where we use events to represent different states of the same locations. We call it LUC Calculus.

\section{THE LUC CALCULUS FORMALISM}

We consider that an interval-based approach is well suited to represent land use change trajectories since changes in land use or land cover are not instantaneous, or at least they can not be observed and measured instantaneously. The conversion of several hectares of forest for be used for agriculture, for example, requires time and money. Given the effort required for this a human-induced transition to be taken place in a given area, this area, in turn, is likely to stay immutable for years. These considerations led us to adopt Allen's interval temporal logic as one of the basic components of our formalism [8].

In the LUC Calculus we use a spatial version of the Allen's predicate in order to aggregate the representation of where the changes happen and what changes are observed. The building blocks of the formalism are:

1. a set of spatial locations $\left(L=l_{1}, l_{2}, \ldots, l_{n}\right)$;

2. a set of properties of locations $\left(P=p_{1}, p_{2}, \ldots, p_{n}\right)$, related to the land use, e.g. Is_a_Forest;

3. a set of non-overlapping and sequential time intervals $\left(T=t_{1}, t_{2}, \ldots, t_{n}\right)$;

4. a set of temporal predicates defined by Allen [9];

5. a set of predicates that can be evaluated at every location:

- $H O L D S(l, p, t)$ : which evaluates as true when a property $p$ holds during the interval $t$;

- $R E C U R\left(l, p, t_{i}, t_{j}\right)$ : which evaluates as true when a location holds a property $p$ during two non-continuous distinct intervals $t_{i}$ and $t_{j}$;

- CONVERT $\left(l, p_{i}, t_{i}, p_{j}, t_{j}\right)$ : which evaluates as true when a location holds the property $p_{i}$ during the interval $t_{i}$, property $p_{j}$ during the interval $t_{j}$ and $t_{j}$ is sequential of $t_{i}$;

- $E V O L V E\left(l, p_{i}, t_{i}, p_{j}, t_{j}\right)$ : which evaluates as true when a location holds the property $p_{i}$ during the interval $t_{i}$, property $p_{j}$ during the interval $t_{j}$ and $t_{j}$ is not necessarily immediately sequential of $t_{i}$.

The LUC Calculus should be applied to a set of locations classified according its land use considering a time granularity of observation. It represents the locations land use trajectories during a given study period. Fig. 1 illustrates the land use change trajectory for a location $l$ from 2001 to 2006 , considering one observation per year: is a forest $(\mathrm{F})$ from 2001 to 2002 , used for pasture (P) in 2003, used for soybean cropping (S) from 2004 to 2005, and for cotton cropping (C) in 2006.

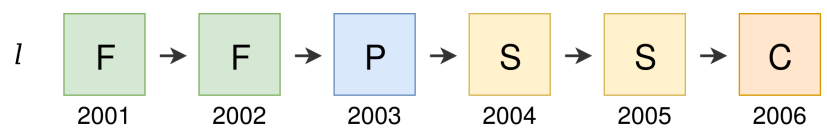

Fig. 1. Example of a land use trajectory in a single location.

Using the components of the LUC Calculus, it is possible to express interesting questions what happened over the study area.

\section{LUC CALCULUS APPLICATION}

To show how the LUC Calculus can be used to reasoning about land use change, we present a case study in Mato Grosso state, Brazil. We selected the municipality of Juruena, in the northeast of the state (see Fig. 2). It has an area of $2,778 \mathrm{~km}^{2}$ and a population of 11,201 inhabitant in 2010 [10].

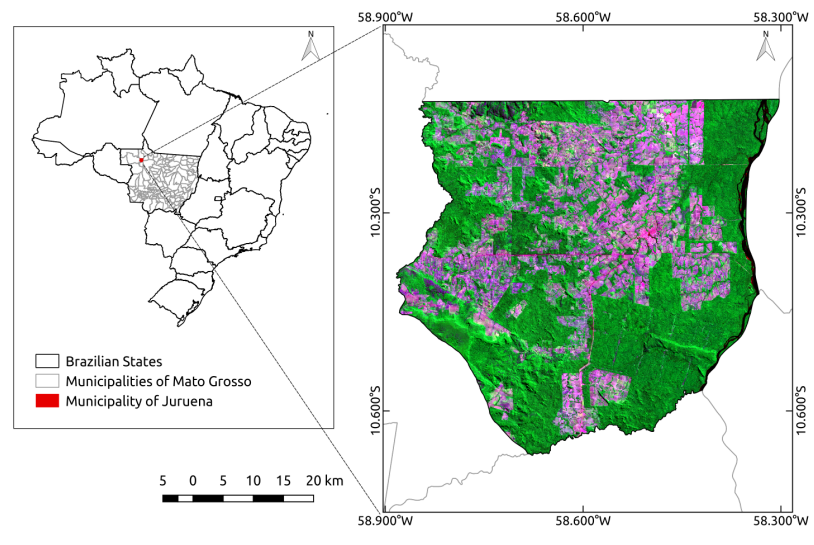

Fig. 2. Juruena study area.

The set of locations are the elements of a regular partition with the resolution of 250 meters, that corresponds to the resolution of MODIS land products used in this case study, resulting in 51,964 locations. For each location its correspondent time series was classified, assigning a land use class (Forest, Degraded Forest (Degradation), Pasture, Water, Single or Double Cropping) for each year during the period from 2001 to 2016. For the classification we used the TWDTW method proposed by Maus et al. [11]. The method aims at finding the best alignments between a set of temporal signatures (or temporal patterns) of a land use classes within a long-term time series of EO data. It uses the length of each pattern as a temporal constraint to produce the best alignments, considering that for example different agricultural crops has distinct phenological cycles. The method performance results directly 
from the quality of the class signatures. In this study case, the class signatures were obtained data collected in the field and also high resolution imagery classified by experts (a total number of 1921 samples).

\subsection{Finding Areas of Secondary Vegetation}

One of our research interests is distinguish secondary vegetation from pristine forest. Pristine forests have not been affected by recent human actions whereas secondary vegetation areas are places where the original forest was cut and the area was later abandoned or unused leading to vegetation regrowth. These areas will appear in remote sensing imagery as forests, despite not having the same characteristics of a pristine forest. However, the growth of the secondary vegetation is seen as an important mechanism to recuperate the regional ecosystem dynamic, so it is important to scientists to be able to identify this areas.

The secondary vegetation class was not directly observed during the classification. Using the LUC Calculus we can express a logical reasoning to uncover the land use transitions leading to the appearing of secondary vegetation areas. In this case study, areas that once were forest, than had other uses before being observed as forest again. Table 1 shows the LUC Calculus expression to represent this reasoning and Fig. 3 shows the annual maps including the locations classified as secondary forest.

Table 1. LUC Calculus expression to select areas of secondary vegetation.

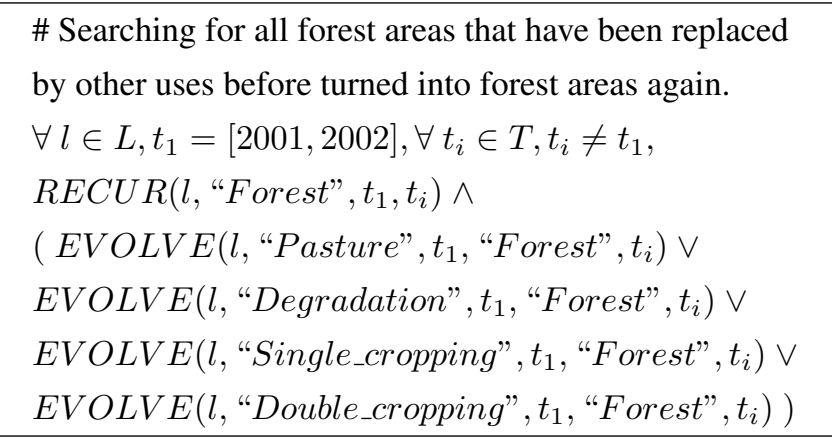

From this results we can quantify the evolution of areas of forest and secondary vegetation during the study period. Fig. 4 shows that the areas of forest decrease during the first years and little secondary vegetation areas observed. After 2010, we can observe an increase in the number of secondary vegetation. Studies show that deforestation for cattle pasture predominated in the northern portion of the Mato Grosso state, where Juruena is located $[2,3]$, our analysis suggest that some a significant part of this locations were not used for grazing and started to regrow.

We compared the amount of deforested areas data produced by the PRODES program. Fig. 5 shows that the input

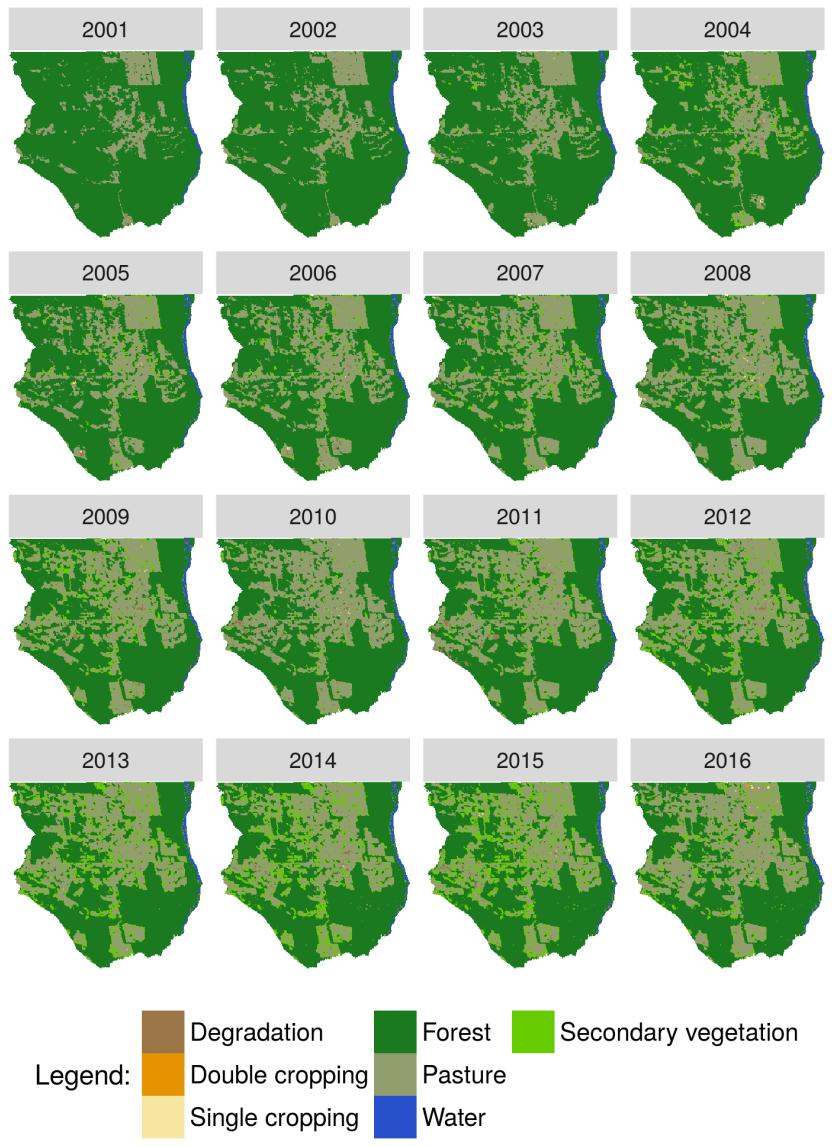

Fig. 3. Land use in Juruena reclassified, from 2001 to 2016.

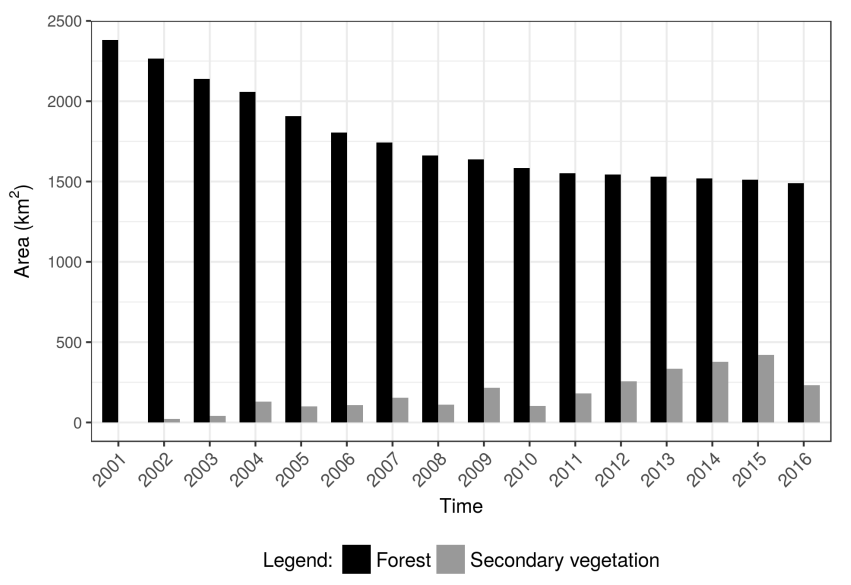

Fig. 4. Total area of forest and secondary vegetation in Juruena from 2001 to 2016.

data used in our application of LUC Calculus overestimates in approximately $30 \%$ the amount of deforestation in the study area between 2002 and 2016, that is equivalent to $280 \mathrm{~km}^{2}$. By dividing this value by the studied years, the error between the classified data and the PRODES data would be equivalent 


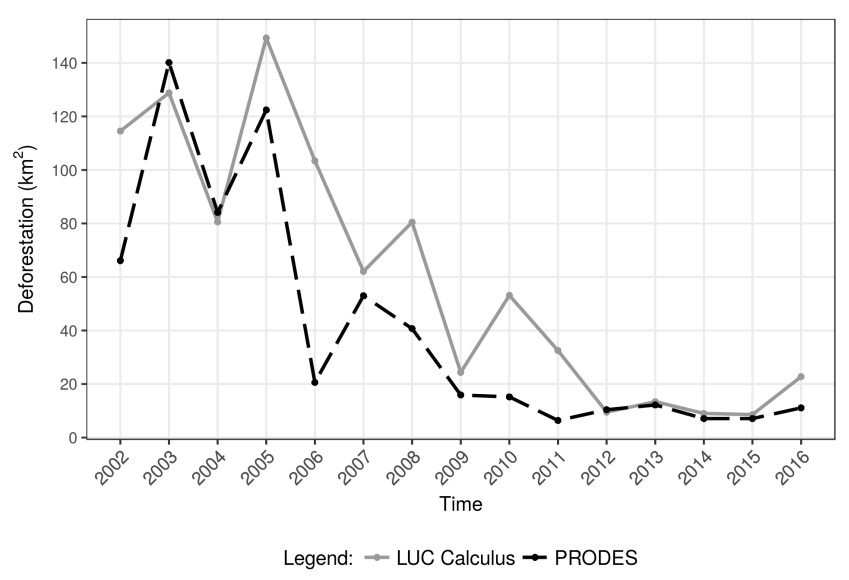

Fig. 5. The total of deforested areas in Juruena, between 2002 and 2016.

to $18.7 \mathrm{~km}^{2}$ per year. This difference results from the spatial resolution used of the data to generate the annual classifications. In our study classified images generated from the analysis of MOD13Q1 product time series from 2001 to 2016, it has with a spatial resolution of 250 meters (MODIS) while PRODES classified images with 30 meters resolution (Landsat class data).

\section{FINAL REMARKS}

This work introduced a spatiotemporal interval logic mechanism, LUC Calculus, for reasoning about land use change trajectories in regional and global areas. We consider discrete transitions where a given land use class is replaced by other one. It allows scientists to express research questions, confirm scenarios and hypothesis and mine and quantify specific data of interest.

Although the formalism proposed is independent of different methods, algorithms and techniques for time series classification from remote sensing data, our approach needs to be supported by a set of consistent and sequential land use trajectories. We believe that long-term time series of EO data can be used to generate classified maps of land use for large areas, with good accuracy and high temporal resolution. However, the quality of the classified maps, used to generate the land use trajectories has a direct influence on the results of the formalism application.

Finally, we have implemented the spatiotemporal interval logic formalism using $\mathbf{R}$, an open source programming language and a statistical environment with a big community of contributors of statistical and graphical packages. This implementation is available at https://github.com/ ammaciel/luccalculus.

\section{REFERENCES}

[1] J. Gil, M. Siebold, and T. Berger, "Adoption and development of integrated crop-livestock-forestry systems in Mato Grosso, Brazil," Agriculture, Ecosystems \& Environment, vol. 199, pp. 394 - 406, 2015.

[2] D. C. Morton, R. S. DeFries, Y. E. Shimabukuro, L. O. Anderson, E. Arai, F. del Bon Espirito-Santo, R. Freitas, and J. Morisette, "Cropland expansion changes deforestation dynamics in the southern Brazilian Amazon," Proceedings of the National Academy of Sciences, vol. 103, no. 39, pp. 14637-14641, 2006.

[3] D. Arvor, V. Dubreuil, M. Simões, and A. Bégué, "Mapping and spatial analysis of the soybean agricultural frontier in Mato Grosso, Brazil, using remote sensing data," GeoJournal, vol. 78, no. 5, pp. 833-850, 2013.

[4] L. Chini, G. Hurtt, S. Frolking, and R. Sahajpal, "LandUse Harmonization (LUH2)," Global Ecology Laboratory, University of Maryland, 2012, Available from: http://luh. umd.edu/index. shtml.

[5] Q. Zhou, B. Li, and A. Kurban, "Trajectory analysis of land cover change in arid environment of China," International Journal of Remote Sensing, vol. 29, no. 4, pp. 1093-1107, 2008.

[6] E. F. Lambin, H. J. Geist, and E. Lepers, "Dynamics of land-use and land-cover change in tropical regions," Annual review of environment and resources, vol. 28, no. 1, pp. 205-241, 2003.

[7] National Institute for Space Research (INPE), "PRODES - Amazon Deforestation Monitoring Project," 2017, Available from: https: //goo.gl/jD1A3b.

[8] J. F. Allen, "Towards a general theory of action and time," Artificial Intelligence, vol. 23, no. 2, pp. 123154, 1984.

[9] J. F. Allen, "Maintaining knowledge about temporal intervals," Communications of the ACM, vol. 26, no. 11, pp. 832-843, 1983.

[10] Brazilian Institute of Geography and Statistics (IBGE), "IBGE - Cities," 2016, Available from: https:// cidades.ibge.gov.br.

[11] V. Maus, G. Câmara, R. Cartaxo, A. Sanchez, F. M. Ramos, and G. R. Queiroz, "A time-weighted dynamic time warping method for land-use and land-cover mapping," IEEE Journal of Selected Topics in Applied Earth Observations and Remote Sensing, vol. PP, no. 99, pp. 1-11, 2016. 